\title{
Políticas públicas para la población en situación de refugiada. Migraciones globales y diálogo intercultural
}

\author{
Public politics for refugees. \\ Global migrations and intercultural exchange
}

\section{Karl Albert Diniz de Souza*}

\footnotetext{
* Profesor y director universitario. Estudiante de posgrado de la Universidad de São Paulo (Instituto de Estudios de Diversidades, Intolerancias, Conflictos, Derechos y Otras Legitimidades [DiversitasUSP]). Investigador sobre diversidad y personas en situación de refugiadas. Activista social a través de servicios de orientación para personas en situación de vulnerabilidad social y afirmación de ciudadanía.

karl.albert@usp.br http://orcid.org/0000-00021611-3312
}

RECIBID O: 15.3.2021

ACEPTAD 0: 17.9.2021

\section{Resumen}

La ciudad global de São Paulo, en el desarrollo de su paradiplomacia, aprobó en plena pandemia, en agosto de 2020, el I Plan de Política Municipal para Inmigrantes, 2021-2024 (PMI). El PMI tiene, al menos en la comunicación pública gubernamental, una marcada orientación hacia la educación popular y el diálogo intercultural. Este ensayo se propone examinar si la educación que se ofrece en la ciudad para la población en situación de refugiada sigue las exigencias de la educación popular, las exigencias de un diálogo entre culturas en situaciones de desigualdad y las exigencias de las personas y los grupos de refugiados. Finalmente, se plantean preguntas para entender si existe o no un diálogo entre culturas en la escuela pública de São Paulo. Las respuestas servirán para comprender si las personas en situación de refugiadas necesitarán fortalecer su capacidad de resiliencia para poder actuar activamente en un futuro cercano, ya que el PMI está orientado a generar evidencia que pueda ser incorporada en la toma de decisiones, especialmente en el período posterior a la actual etapa pandémica.

Palabras clave: migración, refugiado, derechos humanos, Brasil. 


\section{Abstract}

The global city of São Paulo approved the I Municipal Policy Plan for Immigrants 2021/2024 (PMI) in August 2020, amid a pandemic. The PMI is an example of paradiplomacy oriented towards popular education and intercultural dialogue, at least from the local government's public communication perspective. This essay seeks to bring forward contributions to understand whether the education program offered in the city meets the demands of Popular Education, if it fulfils the requirements of dialogue between cultures under unequal situations, as well as the demands of refugee individuals and groups of refugees. Finally, questions are posed to understand whether there is a dialogue between cultures at São Paulo's public schools. The PMI is aimed at generating evidence that can be incorporated into decision-making processes, especially after the current pandemic stage, to give answers to questions such as: will refugees need to strengthen their resilience capacity in the near future and become more active?

Keywords: migration, refugees, human rights, Brazil.

El siglo XXI trae nuevos desafíos y obliga a la sociedad globalizada a repensar su papel. El creciente número de personas en situación de refugiadas (PSR) ${ }^{1}$ exige que la sociedad

En este artículo se utilizará la expresión personas en situación de refugiadas (PSR) para caracterizar a un grupo de población que no se encuentra en su país de origen, que es heterogéneo, compuesto por personas con realidades distintas, aunque compartan la condición de vínculos rotos o debilitados por temores fundados de persecución relacionados con cuestiones de raza, religión, nacionalidad, pertenencia a determinado grupo social u opinión política, así como a violaciones graves y generalizadas de los derechos humanos, eventos climáticos o ambientales que ponen en peligro su existencia o afectan gravemente sus condiciones de vida, incluso por conflictos armados. En el portugués usado en Brasil, sustituir el concepto legal de refugiado por persona en situación de refugiada indica que se valora más la persona que la situación momentánea en la que se encuentra. En el contexto de la construcción de la valoración de los reclamos de ciudadanía y representación social, al utilizar esta expresión, se quiere entender quiénes son las personas en situación de refugiadas y cuál es la razón que las obliga a vivir así. Con la expresión personas en situación de refugiadas el autor pretende crear un espacio de expresión capaz de superar los discursos estigmatizantes y de valorar a la persona que es víctima de refugio obligatorio, objeto de situaciones históricas de exclusión, particularmente en el medio urbano. En este contexto, a las personas en situación de refugiadas les permiten tener su lugar de enunciación y expresión, sin reforzar un lugar social que ocupan, bajo una matriz de dominación y opresión, dentro de las relaciones de poder, que les niegan el acceso a un lugar de pleno ejercicio de la ciudadanía. Para utilizar la expresión personas en situación de refugiadas, el autor brasileño utilizó la metodología de traducción empleada por la editorial española Ediciones Ambulantes, fundada en 2011 en Valladolid con el objetivo de publicar literatura brasileña en español, que en 2020 publicó, por ejemplo, el libro de Djamila Ribeiro Lugar de enunciación, traducido por Aline Pereira da Encarnação y con corrección y revisión de Víctor David López y Carmen Diez. En la lengua portuguesa utilizada en Brasil hay una diferencia significativa entre ser un refugiado y estar en una situación de refugiado. Esta última situación implica dejar de ser un mero objeto de investigación (refugiado) y estar en condiciones de informar y demostrar que su situación no lo/la define completamente como persona, notar el valor de su posición cuando 
internacional busque una reformulación de su forma de actuar, y nos obliga a revisar las relaciones entre las personas y los Estados soberanos. Las relaciones internacionales se desarrollan ahora en un mundo transformado por las revoluciones tecnológicas, por el empoderamiento de los grupos excluidos, por las políticas de afirmación de la ciudadanía y por la acción de nuevos actores, como las empresas privadas y las entidades subnacionales. En este contexto, es necesario comprender si hay un diálogo entre culturas en el contexto de las PSR y en el marco de la paradiplomacia de São Paulo.

La ciudad de São Paulo es el centro financiero, corporativo y comercial de América del Sur, y es la más poblada de Brasil y de todo el hemisferio sur; eso la convierte en la ciudad brasileña más influyente en el escenario mundial. Es la octava más poblada del planeta y su región metropolitana, con unos 21 millones de habitantes, es la décima aglomeración urbana más grande del mundo. En 2019, São Paulo tenía residentes nativos de 196 países, lo que refuerza la necesidad de contar con una política orientada hacia las PSR.

En la situación actual no cabe duda del camino a seguir. Necesitamos caminar con una clara preocupación por proteger a las personas, dando pasos firmes ante la incertidumbre. En este momento hay un letargo de las políticas públicas, que compromete las formas de supervivencia de PSR en varias regiones del mundo.

Durante todo el período inicial de la pandemia y hasta ahora, Brasil, a difer encia de Estados Unidos, ha vivido un gobierno nacionalista y ultraconservador sin una política pública clara sobre la protección de las personas en situación de refugiadas. Sin embargo, la ciudad global de São Paulo viene desarrollando desde hace algunos años su paradiplomacia y aprobó, en plena pandemia, en agosto de 2020, el I Plan de Política Municipal para Inmigrantes (Secretaria Municipal de Direitos Humanos e Cidadania [SMDHC], 2020), trienio 2021-2024 (PMI).

Los pasos futuros dependen del reconocimiento de lo que ha ido sucediendo, principalmente de una reflexión sobre los sucesivos errores que se están cometiendo. Nada se puede resolver con relación a las políticas públicas sin notas críticas sobre el presente y reflexiones técnicas y científicas que formen parte de las acciones necesarias para el futuro de la humanidad, especialmente en las políticas orientadas a las PSR. Según Walsh (2009, p. 42), en este contexto es necesario evocar la llamada interculturalidad crítica, que va más allá del simple reconocimiento de las diferencias y cuestiona las causas de la asimetría cultural y social, buscando reprimirlas. Para el autor: «la interculturalidad crítica debe ir acompañada de una perspectiva decolonial y, por tanto, radicalmente diferente del multiculturalismo liberal».

Dirigentes políticos de diversos países y de las más variadas matrices ideológicas se resisten a admitir, y aún no han logrado entender que la actual crisis humanitaria no

se enuncia el discurso, demostrar que puede hablar por sí mismo, sin necesidad de que alguien lo/la represente o que hable en su nombre (persona en situación de refugiada). 
reconoce fronteras ni espacios geográficos delimitados. Las políticas públicas no pueden permanecer en el dilema entre salvar la economía o salvar a los ciudadanos, o entre mantener medidas de aislamiento o salvar puestos de trabajo. Se trata de una situación que tiene que ver con la creación de nuevas formas de influencia y con la propia naturaleza de la acción descentralizada de los nuevos procesos de la sociedad global.

El desempeño de la ciudad de São Paulo, en el marco de su paradiplomacia, está a la vanguardia de nuevas acciones dirigidas a diversificar roles y actores en la gobernanza global, así como a abordar los asuntos relacionados con las PSR. En este sentido, la ciudad aprobó, el 8 de noviembre de 2020, en plena crisis provocada por la pandemia de covid-19, su primer PMI (SMDHC, 2020).

El PMI es el instrumento jurídico y político de planificación, implementación, seguimiento y evaluación de la política de la ciudad de São Paulo dirigida a la población de otras nacionalidades que residen en su territorio. Se divide en ocho ejes, que comprenden 80 acciones que deben atender las necesidades reales de integración de la población refugiada y migrante que vive en su territorio.

Cabe señalar que desde 2016 la ciudad de São Paulo cuenta con una política para la población refugiada (ley 16.478, Brasil, São Paulo, 2016), y la acción y los esfuerzos simultáneos hacen que el PMI y el Pacto Mundial sobre Refugiados estén en total armonía y complementariedad. Según datos de UNHCR/ACNUR (United Nations High Commissioner for Refugees, 2021), más de 360.000 refugiados y migrantes de alrededor de 200 nacionalidades viven en São Paulo.

La ley 16.478 (Brasil, São Paulo, 2016), que instituye la política educativa de la ciudad de São Paulo para la población inmigrante, garantiza a todos los niños, niñas, adolescentes, jóvenes y adultos inmigrantes el derecho a la educación, mediante el ingreso, la permanencia y la terminación en la red local de educación pública. Para el ejercicio de este derecho no constituye obstáculo la imposibilidad de acreditación documental de los años escolares ya cumplidos.

Según Tavares y Gomes (2018, p. 59), la situación de las migraciones y la configuración de las poblaciones de refugiados son un fenómeno creciente a escala mundial que plantea un gran desafío en lo que respecta a la reconfiguración social resultante de la «inclusión» de personas en diferentes culturas. Para los autores:

[...] las discusiones y los debates que tienen lugar en el mundo académico, y fuera de él, sobre el multiculturalismo se justifican por el hecho de que durante siglos ha estado ausente cualquier referencia a la diversidad cultural y al multiculturalismo, ocultas por el monoculturalismo. (Tavares y Gomes, 2018)

Para comprender la propuesta de PMI para el diálogo intercultural, es necesario comprender su contexto teórico. Para Walsh (2013, p. 47), la práctica pedagógica del 
diálogo intercultural consiste en el conjunto de prácticas, estrategias y metodologías con las que se fortalece la construcción de resistencias e insurgencias: "Además de los espacios escolares y el sentido instrumentalista de enseñanza y transmisión de conocimientos, estas prácticas se ponen al servicio de las luchas de liberación social, política, ontológica y epistémica».

La ley 16.478 (Brasil, São Paulo, 2016), en su artículo 20, define que la educación observará el principio de interculturalidad, promoviendo el diálogo entre las diferentes culturas, la ciudadanía democrática y una cultura de paz. Al respecto, el Gobierno de la ciudad de São Paulo es responsable de:

I. Priorizar y ampliar las acciones educativas para combatir la xenofobia, considerando sus interfaces con otras formas de discriminación;

II. Introducir contenidos que promuevan la interculturalidad y la valoración de las culturas de origen de los estudiantes inmigrantes o hijos de inmigrantes dentro del plan de estudios, en todas las asignaturas y etapas de la educación, con la inclusión de materiales didácticos sobre la temática de las corrientes migratorias contemporáneas, incluidos el refugio y el diálogo intercultural;

III. Fortalecer y ampliar los programas de formación intercultural dirigidos a los profesionales de la enseñanza;

IV. Promover, difundir y garantizar el apoyo pedagógico, material e institucional a proyectos de acogida, promoción de la interculturalidad y valoración de la cultura de origen de los estudiantes inmigrantes y sus familias, con su participación en los establecimientos educativos y equipamientos públicos municipales en general.

De todos modos, lo que pretendemos entender es si el desempeño de la ciudad global de São Paulo en educación intercultural es una macrotendencia (cambio a gran escala que afecta a diferentes segmentos de la sociedad) o una microtendencia (cambio de corto plazo que afecta a un número menor de personas). Los resultados podrán definir si habrá una acción, o una reacción, ante las demandas globales de tratamiento de la población migrante, especialmente en lo que respecta al PSR. Sin embargo, como observa Walsh (2013, p. 48), la interculturalidad debe entenderse como un proyecto político antihegemónico propuesto y asumido en primer lugar por los propios estudiantes y, sobre todo, incluir las prácticas educativas liberadoras de los más populares sectores de la sociedad brasileña, como las PSR, «entendiendo que el camino de la interculturalidad crítica es una forma de romper el modelo de pensamiento único responsable de excluir el universalismo», a diferencia del modelo propuesto por el neoliberalismo, que se configura como una microtendencia.

Para Tubino (2005, p. 39), es importante observar los datos pasados y actuales para comprender qué patrón forman. A partir de este supuesto teórico, es posible predecir los 
comportamientos que probablemente se establecerán en el futuro, en relación con el desempeño del Gobierno de la ciudad de São Paulo, en lo que respecta a la educación popular y el diálogo intercultural y las respectivas prácticas orientadas a la RSP. Para el autor, la interculturalidad no es un concepto, es una forma de comportamiento. No es una categoría teórica, es una propuesta ética: «Más que una idea, es una actitud, una forma necesaria en un mundo paradójicamente cada vez más interconectado tecnológicamente y al mismo tiempo más incomunicable interculturalmente». Así, la interculturalidad crítica funciona como una posibilidad de diálogo sobre territorialidades y PSR.

La Secretaría de Derechos Humanos y Ciudadanía, así como la Secretaría de Educación, trabajan de manera integrada para atender las demandas educativas de las PSR en la ciudad de São Paulo. Las acciones aprobadas para las PSR se pueden dividir en dos áreas principales: cursos de educación en derechos humanos y cursos de portugués para la población inmigrante. El trabajo desarrollado durante las últimas administraciones de la ciudad de São Paulo ha mantenido, desde la creación de la Secretaría de Derechos Humanos, en 2015, la formación en derechos humanos y el curso de lengua portuguesa y cultura brasileña. Durante la crisis humanitaria del covid-19, las acciones se adecuaron al modelo virtual o semipresencial, cuando fue posible esta modalidad.

Las PSR reciben acciones del Centro de Referencia y Atención al Inmigrante (CRAI) y de los Centros de Educación en Derechos Humanos. El CRAI trabaja ofreciendo apoyo especializado y multilingüe a los inmigrantes, enfocado en la orientación en regularización migratoria, el acceso a derechos sociales, la orientación sobre servicios legales y sociales, el envío de denuncias por violaciones de derechos humanos, y la oferta de formación en lengua portuguesa y cultura brasileña. Este curso para inmigrantes y refugiados responde a la demanda de quienes necesitan aprender portugués rápidamente, no solo para la expresión cotidiana, sino también para las cuestiones formales que permiten obtener documentación y trabajo.

Para Bittar (2016, p. 23), los Centros de Educación en Derechos Humanos (CEDH) son polos permanentes de promoción de la educación en derechos humanos y fomento de prácticas ciudadanas, basados en la red de educación y articulación con los territorios de la ciudad de São Paulo, que permiten también acciones para las PSR.

Según Bittar (2016, p. 29), a partir de cinco frentes de acción (cultura de participación, articulación territorial, material formativo y pedagógico, acervo de derechos humanos y calendario de actividades culturales y educativas) los Centros de Derechos Humanos funcionan como espacios de convergencia de acciones e iniciativas para las PSR realizadas en el territorio. Su punto de partida es la escuela, que se constituye como un locus privilegiado para promover una educación basada en valores y prácticas de ciudadanía.

Este sigue siendo el escenario de las escuelas públicas de la ciudad de São Paulo en lo que respecta a la enseñanza de los derechos humanos para las PSR, en el marco del PMI. En este contexto, destaca el rol que cumplió Paulo Freire como secretario de 
Educación en la ciudad de São Paulo (1989-1991), que en ese momento reforzó el lugar de la educación continua en las políticas públicas. Freire teorizó y experimentó la formación docente, a la que consideró una necesidad de formación permanente.

Para Tavares y Gomes (2018, p. 60), las pedagogías interculturales deben tener sus reflejos en las instituciones educativas como resultado de prácticas pedagógicas construidas en espacios sociales y escenarios de lucha y resistencia. Según los autores, en el contexto actual del PMI, «la propuesta pedagógica de sensibilización de Freire gana cada vez más sentido».

Según Freire (2006, p. 63), la formación es fundamental para cualquier desarrollo de la acción pedagógica, especialmente para la práctica de la educación popular. Como secretario de Educación y responsable de la implementación de las políticas públicas, Paulo Freire se anticipó al debate intelectual e implementó durante su mandato la «formación continua» en las escuelas de la ciudad. Esta decisión produjo buenos resultados y sus efectos se pueden sentir aún hoy, pero en los últimos veinte años se ha alentado poco. La formación para la educación popular y para el diálogo intercultural ha reaparecido en ciertos momentos, pero no se ha consolidado como una realidad permanente.

Solo durante la gestión de Eduardo Suplicy como secretario de Derechos Humanos y Ciudadanía (2015-2016) se retomó en la ciudad de São Paulo la preocupación por la formación de docentes en el área de derechos humanos, con la metodología de la educación popular. Suplicy $(2015$, p. 31) demuestra la preocupación por vincular los dos temas y la participación del Instituto Paulo Freire en este contexto:

El propósito de esta publicación es permitir una mejor comprensión de las relaciones entre derechos humanos, ciudadanía, educación popular, participación social, derecho a la ciudad, además de presentar formas más participativas y herramientas de gestión adoptadas por la actual administración.

El trabajo de Freire es sumamente actual, sobre todo en lo que respecta a la aplicación de la educación a las PSR. Para Freire (2015, p. 64), la educación debe estar relacionada con la lectura del contexto del mundo, en la que estudiantes y docentes deben ser actores activos que fomentan las distintas identidades culturales con vistas a superar las estandarizaciones propias de una práctica pedagógica dominante.

A partir de estos valores, notamos que los principios de Paulo Freire siguen siendo defendidos en las actividades de educación en derechos humanos. Los directores han logrado, a pesar de las limitaciones institucionales, entablar una relación con los estudiantes basada en una educación plena y liberadora, en la que los niños y jóvenes no son «depósitos de contenido», sino que pueden convertirse en ciudadanos más participativos. El PMI procura desarrollar la educación en derechos humanos para las PSR, con el 
apoyo de la escuela y la familia, que es, de hecho, el primer entorno educativo. Específicamente en la ciudad de São Paulo, educar en derechos humanos, principalmente cuando se trata de las PSR, corresponde a integrar la vida escolar en la vida cotidiana de los niños, niñas, jóvenes y la comunidad en la que viven.

\section{Educación popular y diálogo intercultural para las PSR: entre puentes y muros}

Las PSR que llegan a la ciudad global de São Paulo viven una realidad compleja, en la que necesitan superar muchos muros (xenofobia, racismo, burocracia, falta de agilidad en los procesos de legalización de sus respectivas situaciones), pero, al mismo tiempo, pueden encontrar puentes (apoyo de organismos sociales, humanitarios y públicos, así como voluntarios que brindan refugio, a diferencia de la realidad del país que dejaron atrás).

Entre puentes y muros, las PSR encuentran situaciones vinculadas a la educación que plantean muchas preguntas. ¿Cómo el refugiado recibe acceso a la educación y qué tipo de educación recibe, así como cuál es el modelo pedagógico que utilizan los educadores y voluntarios para contactarlos y con ellos? En la concepción de Freire, cabe cuestionar lo que ocurre en la ciudad de São Paulo en cuanto a la educación ofrecida, no solo en el «espacio físico», sino también en el «espacio histórico dialéctico». El flujo de PSR a São Paulo se ha incrementado en los últimos años, pero ¿cómo se incorporó las PSR al sistema educativo oficial?

Para Azibeiro y Fleuri (2012, p. 219), actuar en el espacio del diálogo intercultural no significa no tomar posición. Por el contrario, presupone entender que cualquier declaración de neutralidad ya está tomando partido. En este sentido, el encuentro o la confrontación intercultural tiene dos manifestaciones posibles: 1) o se admite la emergencia de lo plural, lo múltiple, lo divergente, o 2) las voces disonantes son reprimidas y excluidas. Para estos autores, la tarea de la educación intercultural no es adaptarse, ni siquiera posibilitar la comprensión mutua de lenguas. Destacan la clara necesidad de actuar para permitir la emergencia de múltiples significados, provocando la reflexión sobre sus flujos y cristalizaciones.

¿Cuál es el lugar de enunciación de las PSR? Esta pregunta se refiere al antagonismo que enfrentan estas personas al llegar a la ciudad de São Paulo: muros y puentes. Ofrecer un discurso a los PSR no implica silenciar a nadie (muros), sino hacer espacio para que se escuchen y consideren diferentes voces (puentes). Con relación al proceso de aprendizaje ofrecido en la ciudad de São Paulo, o al menos propuesto por los organismos públicos, es fundamental definir el lugar de enunciación de las PSR, explicar si 
son protagonistas o no en este contexto. La respuesta necesita articular un aspecto clave que no siempre se percibe en toda su dimensión, que es el diálogo entre culturas en la escuela y en conexión con otras experiencias educativas comunitarias.

Es preciso pensarla en su relación con la cuestión del diálogo entre culturas en el contexto de las PSR y en el marco de la paradiplomacia en la ciudad de São Paulo. Para comprender esta situación, Walsh (2013, p. 42) encuentra en Paulo Freire y Frantz Fanon, por su sentimiento pedagógico y político, a dos intelectuales comprometidos con las luchas por la liberación y la descolonización, que han hecho importantes aportes a la educación crítica intercultural y descolonial. Esta autora señala dos puntos de partida complementarios (el pedagógico en Freire y el problema colonial en Fanon), que proponen, ambos, la lucha decolonial, de liberación y de humanización:

El punto de partida de Fanon fue el problema colonial; describió y narró la situación de la colonización y promovió y reveló la lucha anticolonial. Freire, en cambio, partió de la problemática de la opresión: las condiciones existenciales de las clases pobres y excluidas por el orden injusto, en cuyo contexto la violencia de los opresores deshumaniza a los oprimidos. Si bien Freire se ha enfocado más intensamente en el aspecto pedagógico, ambos contribuyen efectivamente a una propuesta que articula lo político y lo pedagógico con la educación intercultural crítica y decolonial, con miras a un sentido práctico y concreto en favor de las luchas de descolonización, liberación y humanización.

Para Azibeiro y Fleuri (2012, p. 241), se operacionaliza como una pedagogía de encuentro-confrontación llevada a sus últimas consecuencias. Esta comprensión de la educación intercultural enfatiza la relación entre sujetos (individuales y colectivos), buscando posibilitar «una producción efectivamente plural de significados y lugares mediante la comprensión de que los significados se pueden reelaborar en los procesos de interacción social, estableciendo contextos relacionales que inventan otras políticas».

Con relación al PMI, es fundamental cuestionarse si la educación que se ofrece a las PSR es una educación popular e intercultural que enfrenta el colonialismo intelectual, el tradicionalismo pedagógico y el autoritarismo de la ciencia tradicional europea. ¿Se alienta a las PSR a producir un pensamiento científico y pedagógico que rompa con la subordinación de conocimientos y experiencias de sujetos sociales marginados? ¿Existe una construcción colectiva de metodologías y propuestas didácticas que hagan posible la participación y la construcción del conocimiento apoyándose en la acción activa de las PSR? La educación en la ciudad de São Paulo para las PSR ¿refleja un proyecto de emancipación que garantiza el derecho de todas las personas a la existencia, el trabajo, la libertad, la justicia y la educación transformadora? 
La educación que se ofrece a las PSR ¿sigue los moldes de una educación popular o de una educación tradicional? Para caracterizar la primera opción, el PMI debe cumplir algunos requisitos fundamentales. Uno de ellos es contribuir, a través de la educación, al empoderamiento de las PSR para que puedan enfrentar las diferentes formas de opresión y ser parte de una sociedad local más solidaria e inclusiva. En este contexto, el diálogo entre culturas en la escuela y en relación con otra educación comunitaria deberá reducir el impacto de la crisis social sobre la pobreza, agravada por la pandemia de covid-19, y dar voz a las PSR en la ciudad de São Paulo.

Para analizar si el PMI desarrollará una educación popular e intercultural, los actos y prácticas de política pública deben tener al menos tres características enumeradas por Brandão (1994, p. 49). La primera característica es que la educación popular necesita una práctica renovadora en las relaciones humano-sociedad-cultura-educación y una acción pedagógica, basada en estas relaciones, que genere una educación liberadora. La segunda característica es que la educación no sea solo una actividad formal de escolarización popular, alfabetización, sino que logre convertirse en una práctica sistemática de intercambio de conocimientos a partir de las prácticas sociales de las propias PSR. Finalmente, la educación popular no puede dejar de lado la labor política de mediación al servicio de proyectos, sujetos y movimientos populares de PSR, con vistas a construir un nuevo rol dentro de la sociedad capitalista dependiente.

La ley que instituyó el PMI propone prácticas pedagógicas de base popular, ya que buscan construir una educación orientada a las PSR, en una escuela viva, basada en la interacción de las diferencias, la transformación de espacios, el análisis crítico de la realidad y la inclusión de los debates multiculturales de estas personas, así como la comprensión de las diferencias y que estas no representan inferioridad. Al analizar las diferencias entre Freire y Fanon, Walsh (2013, p. 51) declara:

Si bien Freire propone que, para enfrentar la opresión y llegar a la humanización, es necesario conocer la realidad para transformarla, Fanon sugiere que el peso corporal de la colonialidad y la consecuente exterioridad conducen a una nueva forma de conocer cómo el sujeto colonizado interioriza su proceso de colonización, con lo que crea las condiciones de inexistencia.

Asimismo, la política de la ciudad de São Paulo para la enseñanza de los derechos humanos, basada en el diálogo entre culturas en la escuela misma y en vínculo con otras educaciones comunitarias, orienta sus lineamientos a construir un currículo colectivamente, con el objetivo de valorar la diversidad cultural y la formación crítica, buscando superar las exclusiones, ya sea de género, raza, etnia, orientación sexual o territorialidad. Para practicar una educación popular e intercultural para las PSR es necesario volver a los términos de Freire y entender esta comunidad popular como un grupo social 
de oprimidos. Así, las PSR son oprimidas y populares, ya que viven sin las condiciones elementales para ejercer su ciudadanía activa.

El PMI propone un tratamiento diferenciado con relación a las PSR (adultos y niños). A los adultos se les respeta su historia personal en cuanto a conocimientos previos y reciben clases de portugués distintas a las de los niños. Por su condición de PSR, no fue su elección vivir en un país donde se habla otro idioma, sino una necesidad. Por eso, las clases de lengua portuguesa y cultura brasileña en las escuelas públicas deben ser ofrecidas como portugués como lengua anfitriona (PLAc en portugués), que tiene una metodología propia y respeta la identidad de quienes la están aprendiendo.

En este contexto de educación popular, el PMI establece en la propuesta de acción 66, ítem 4: «Fortalecimiento de la política municipal de acogida de estudiantes inmigrantes mediante la contratación de profesores itinerantes de portugués como lengua anfitriona (PLAc) para trabajar en escuelas con concentración de estudiantes inmigrantes» (SMDHC, 2020).

De acuerdo con el trabajo de Freire, también existe preocupación por la formación continua de los profesores que trabajan en la enseñanza de idiomas para PSR. El PMI para la población inmigrante de São Paulo establece en propuesta de acción 67, ítem 3: «Mejorar el Programa Puertas Abiertas a través de la formación continua de profesores de portugués como lengua anfitriona (PLAc), fomentando la contratación de profesores inmigrantes y proponiendo alianzas con universidades» (SMDHC, 2020).

En términos basados en Freire, al respetar la cultura de las PSR y prever la posibilidad de contratar a profesores inmigrantes para continuar el proceso de incorporación de estas personas mediante el aprendizaje de la lengua nacional, se implementa una educación popular, capaz de traspasar los límites escolares para buscar el rescate de ciudadanía, el rescate de la dignidad individual y la necesidad de inclusión en todos los sentidos.

Los últimos grandes flujos migratorios internacionales han ampliado la realidad de la ciudad global de São Paulo y han demostrado la necesidad de nuevas acciones que permitan una mejor integración de la población migratoria. El PMI afirma que la oferta de cursos destinados a la enseñanza de la lengua portuguesa debe ser tratada como un instrumento de política de acogida de inmigrantes y refugiados. Debido a la falta de material didáctico específico de PLAc, utiliza en sus cursos el material didáctico difundido por la Agencia de la ONU para los Refugiados (ACNUR) en alianza con entidades tradicionales que trabajan con PSR. El título de este folleto sobre la enseñanza de portugués específicamente para las PSR en Brasil es: Puede ingresar: Portugués brasileño para refugiadas y refugiados, y se obtiene de forma gratuita en el sitio web de la Secretaría de Derechos Humanos y Ciudadanía de la ciudad de São Paulo.

Oliveira et al. (2021, p. 5) demuestran que existía una preocupación por incluir y vincular, en términos basados en el trabajo de Freire, las PSR en la elaboración del libro: 
Hicimos una valoración con PSR antes, para encontrar cuáles eran sus demandas principales, sus principales dificultades en Brasil, y estábamos tratando de crear un orden de prioridad en los contenidos, que se irán incrementando en complejidad a medida que aumente el vocabulario.

Asimismo, afirman:

Lo que encontramos es un mercado con material educativo para un público específico. Está dirigido a turistas, enseña «cómo pedir un taxi en la avenida Paulista». Entonces no es algo que se ajuste a la realidad de la vida de las refugiadas y refugiados. (Oliveira et al., 2021)

En este contexto de educación popular y valoración del diálogo intercultural, el PMI establece, en la propuesta de acción 1, que el Gobierno «reconoce a estas personas como sujetos de derecho y trabajará desde la perspectiva de género, interculturalidad e interseccionalidad, de manera descentralizada» (SMDHC, 2020).

Sin embargo, a pesar de que existe una disposición legal sobre el diálogo intercultural, es necesario recordar las preocupaciones de algunos autores sobre este tema. Para Azibeiro y Fleuri (2012, p. 241), la perspectiva del diálogo intercultural no debe tener posturas misioneras, que arrojan acciones e investigaciones en torno a lo popular a otro nivel. «En el pasado la cultura dominante hacía imposibles algunas de las aspiraciones de dignidad humana de la cultura subordinada. Ahora será posible pronunciarlas en el diálogo intercultural, y hacerlo sin justificar o incluso reforzar la subordinación». Si bien en su concepción existe una preocupación por el diálogo intercultural, es necesario verificar si ello realmente se llevará a cabo en la ejecución del PMI.

Entender al otro es esencial para que cualquier propuesta sirva correctamente a las personas que abandonaron su tierra. El idioma es la puerta de entrada al desafío de vivir en un nuevo país. La enseñanza del portugués con la preocupación de que se base en las necesidades de las PSR es un indicio de que el PMI está orientado a generar evidencias que se puedan incorporar a la toma de decisiones.

\section{Conclusión}

¿Acción o reacción? La acción, o reacción, surgirá de la conciencia, o no, de que la migración no puede ser gestionada y controlada eficazmente, exclusivamente, por las políticas migratorias nacionales.

La paradiplomacia en la ciudad de São Paulo es un ejemplo real de un fenómeno de creciente importancia en las relaciones internacionales: la acción diplomática de los 
gobiernos subnacionales. ¿A qué conclusión podemos llegar sobre la tendencia actual del Gobierno de la ciudad de São Paulo en el desarrollo de la educación popular y el diálogo intercultural para impulsar acciones de educación para la RSP? Podemos entender que el Gobierno de la ciudad, que está proponiendo estos temas, tendrá acceso a información valiosa sobre el comportamiento de los involucrados, así como la posibilidad de crear una estrategia sobre el tema capaz de estar a la vanguardia.

En cualquier caso, este problema tiene que ver con la creación de nuevas formas de influencia y con el carácter muy descentralizado de los nuevos procesos de gobernanza global. Las acciones realizadas hasta el momento solo indican que los efectos de la emergencia sanitaria de covid-19 causarán un fuerte impacto en la población inmigrante. Cabe destacar que las PSR deben estar preparadas para enfrentar escenarios más restrictivos en cuanto a su circulación, así como a su asentamiento, aunque sea temporal, en territorios distintos a su lugar de residencia original.

Este artículo solo analizó la educación desarrollada para las PSR en escuelas públicas de la ciudad de São Paulo. No es un diagnóstico del sistema educativo oficial. Sabemos que no existe una propuesta exclusiva para la enseñanza de los derechos humanos a las PSR, pero en las escuelas públicas locales todos los niños y jóvenes, incluidas las PSR, pueden participar en las actividades. No hay un proyecto exclusivo para las PSR. Se garantiza su derecho fundamental de acceso a las instalaciones públicas y escuelas en la red pública, lo que requiere reforzar los temas de inclusión, diálogo intercultural y la flexibilidad en la aplicación de las reglas burocráticas.

La oferta de cursos de lengua portuguesa y cultura brasileña no está dirigida solo a las PSR, sino a toda la población inmigrante. Dentro de las posibilidades de ofrecer clases durante 2020 y principios de 2021 (falta de un lugar de estudio y de dispositivos adecuados, mala conexión a internet, falta de material de apoyo impreso y digital, así como de formación para que los docentes trabajen de forma aislada) se ofrecieron actividades. Sin embargo, es necesario repensar las acciones para las PSR en la ciudad de São Paulo, a fin de que brinden una educación diseñada para el acceso igualitario (cultural, político y tecnológico) en situaciones más seguras e inclusivas que las que existían antes de la pandemia.

Las PSR deberán fortalecer sus capacidades de resiliencia frente a escenarios futuros y eventos inesperados que probablemente se presenten en un futuro próximo. En todo caso, es fundamental que la cuestión del diálogo entre culturas en el contexto de las PSR y el marco de la paradiplomacia en la ciudad de São Paulo se mantenga y amplíe en los próximos años. El desafío del diálogo entre culturas en la escuela y en relación con otras educaciones comunitarias, en el marco del PMI, requiere una reflexión permanente. Se deben observar las políticas públicas orientadas a las PSR, en especial las prácticas educativas desarrolladas para producir nuevos caminos pedagógicos con una di- 
mensión intercultural y de educación popular. Por lo tanto, no basta incluir esos conceptos teóricos en la ley que instituyó el PMI, sino que se requiere una práctica pedagógica comprometida y permanente, que aún está en proceso de implementación en la ciudad de São Paulo.

\section{Referencias bibliográficas}

Azibeiro, N., y Fleuri, R. (2012). Paradigmas interculturais emergentes na educação popular. En S. Dantas (org.), Diálogos interculturais: Reflexões interdisciplinares e intervenções psicossociais (pp.219-247). São Paulo: Universidade de São Paulo.

Bittar, E. (2016). Educação em direitos humanos da cidade de São Paulo: Territórios, educação e cidadania. São Paulo: Coordenação de Educação em Direitos Humanos.

Brandão, C. (1994). Os caminhos cruzados: Formas de pensar e realizar a educação na América Latina. En M. Gadotti y C. A. Torres (eds.), Educação popular: Utopia Latino-Americana. São Paulo: Universidade de São Paulo.

Brasil, São Paulo. (2016). Lei 16.478 de 08 de julho de 2016. Recuperado de http://legislacao.prefeitura.sp.gov.br/leis/lei-16478-de-08-de-julho-de-2016/.

Freire, P. (2006). A educação na cidade. (7. a ed.). São Paulo: Cortez.

Freire, P. (2015). Pedagogia da autonomia. Rio de Janeiro: Paz e Terra.

Oliveira, T., Reinoldes, M., Mandalá, P. S., y Amado, R. S. (2021). Pode entrar: Português do Brasil para refugiadas e refugiados. Recuperado de https://www.prefeitura.sp.gov.br/cidade/secretarias/direitos_humanos/imigrantes_e_trabalho_decente/programas_e_projetos/portas_abertas/index.php?p=259310.

Secretária Municipal de Direitos Humanos e Cidadania. (2020). I Plano municipal de políticas para imigrantes. São Paulo: SMDHC.

Suplicy, E. (2015). Comitê Municipal de Educação em Direitos Humanos. São Paulo: Instituto Paulo Freire. (Cadernos de Colegiados).

Tavares, M., y Gomes, S. R. (2018). Multiculturalismo, interculturalismo e decolonialidade: Prolegômenos a uma pedagogia decolonial. Dialogia, 29, 47-68.

Tubino, F. (2005). Del interculturalismo funcional al interculturalismo crítico. Recuperado de http://red.pucp.edu.pe/wp-content/uploads/biblioteca/inter_funcional.

United Nations High Commissioner for Refugees. (2021). Refugee data finder. Recuperado de https://www.unhcr.org/refugee-statistics.

Walsh, C. (2009). Interculturalidad, Estado, sociedad: Luchas (de)coloniales de nuestra época. Quito: Universidad Andina Simón Bolívar.

Walsh, C. (2013). Pedagogías decoloniales: Prácticas insurgentes de resistir, (re)existir y (re)vivir. Tomo I. Quito: Universidad Andina Simón Bolívar. 